\title{
28 Research Square \\ Remote clinician-based home management of COVID-19 associated pneumonia in a resource limited setting
}

Nadir Kana

University of the Witwatersrand https://orcid.org/0000-0001-8902-2684

Shivesh Maharaj ( $\square$ shivesh.maharaj@wits.ac.za)

University of the Witwatersrand https://orcid.org/0000-0002-2118-2400

\section{Case Report}

Keywords: COVID19, Sars-COV-2,remote patient management, home-based care

Posted Date: July 24th, 2020

DOI: https://doi.org/10.21203/rs.3.rs-47965/v1

License: (c) (1) This work is licensed under a Creative Commons Attribution 4.0 International License.

Read Full License 


\section{Abstract}

The coronavirus disease (COVID-19) pandemic has resulted in an increased number of patients that require respiratory support in terms of oxygen and ventilation. Due to the burden on the healthcare system there has been a shortage of hospitals beds in both the public and private sector. This has led to many patients being managed at home. Currently there is no consensus regarding best practice guidelines in terms of treatment of this subset of patients.

We hereby present a case of a patient who was treated using appropriate respiratory support (a combination of low flow oxygen therapy via nasal prongs or face mask as high flow oxygen was not available), oral corticosteroids and anti-coagulants managed by remote clinician intervention. We also include our home management protocol.

\section{Background}

COVID-19 is caused by a new strain of Coronavirus (SARS-CoV-2) discovered in Wuhan, China where the first cases where reported in December 2019. On 11 March 2020 the World Health Organization (WHO) declared the current COVID-19 outbreak a public health emergency of global concern and ultimately a pandemic. $^{1}$

With the rapid rise in COVID-19 cases, hospitals worldwide are reporting shortages of essential resources namely intensive care unit (ICU) beds, medical equipment and personal protective equipment (PPE) which is placing pressure on the health care system. Many institutions have postponed and/or cancelled elective and non-urgent procedures to conserve resources and limit exposure. ${ }^{1,2}$

In South Africa, the critical care capacity and resources of hospitals, in particular, ICU/High care beds and high flow oxygen devices are constrained and poses a great challenge within the current COVID-19 scenario and is placing immense pressure on the provision of healthcare. Patients with respiratory failure require much higher concentrations of oxygen that can be achieved by specialised high flow nasal cannula devices or mechanical ventilation. ${ }^{3}$

Healthcare practitioners are seeking alternative solutions to safely manage patients at home, even those with COVID-19 pneumonia. With the abovementioned constraints and resource limitations, the possibility of remote clinician based home management of patients with COVID-19 associated pneumonia should be considered.

\section{Case Presentation}

In June 2020, a 41-year-old female nursing manager with a history of chronic rhino-sinusitis presented with myalgia, headache and a postnasal drip with no documented COVID-19 exposure. The patient consulted with her local general practitioner (GP) who diagnosed acute sinusitis and prescribed amoxicillin, nasal decongestants and analgesics. 
On day 3 , the symptom severity escalated however up until this stage no pyrexia was evident. The patient returned to the GP as her symptoms were not improving and a real-time reverse transcription-polymerase chain reaction (rRT-PCR) nasopharyngeal swab was taken. She was advised to isolate with a clinical diagnosis of COVID-19 infection due to the fact that she was a frontline healthcare worker.

On day 4, pyrexia of greater than $38^{\circ} \mathrm{C}$ and dyspnoea ensued and the patient went to her nearest Accident \& Emergency unit (A\&E unit). No co-morbidities were noted.

A Chest X-Ray was completed (figure 1A) and she was diagnosed with bronchopneumonia.

The rRT-PCR of her nasopharyngeal swab returned positive for SARS-CoV-2 but the patient's clinical condition did not meet the institution's criteria for hospital admission.

\section{TREATMENT}

Since the patient did not meet the hospital admission criteria, she was advised to self-isolate at home and follow a prescribed home care protocol as recommended by the clinician. She was instructed and guided on how to monitor her oxygen saturation and respiratory rate, and to return to the A\&E unit if she de-saturated or if her condition deteriorated. The patient was advised to start Vitamin C, D and Zinc supplementation.

Home oxygen at two litres per minute was initiated via nasal canulae. The patient was placed in a prone position. Oxygen saturation immediately improved to $93 \%$ but the respiratory rate remained persistently high above 20 bpm. Rivaroxaban $10 \mathrm{mg}$ twice daily orally was initiated.

\section{INVESTIGATIONS}

Complete blood count revealed white blood cells, $9.57 \times 10^{9} / \mathrm{L}$ (neutrophils, $42.3 \%$; lymphocytes, 50.6\%; and monocytes, $6.5 \%$ ); haemoglobin $13.4 \mathrm{~g} / \mathrm{dL}$; mean corpuscular volume, $85.7 \mathrm{fL}$; and platelets, $344 \mathrm{x}$ $10^{9} /$ L.

Serum laboratory test results were as follows: creatinine, $0.9 \mathrm{mg} / \mathrm{dL}$; sodium, $143 \mathrm{mmol} / \mathrm{L}$; potassium, 4.0 $\mathrm{mmol} / \mathrm{L}$; chloride, $108 \mathrm{mmol} / \mathrm{L}$; C-reactive protein, $17.8 \mathrm{mg} / \mathrm{L}$; and quantitative D-Dimer, 0.23ug/MI.

Spirometer test was conducted and the Flow Volume Test results indicated a mild restrictive abnormality. A reduced FVC with normal FEV1\% may only suggest restriction Low vital capacity, which is perhaps due to restriction of lung volumes.

\section{DIFFERENTIAL DIAGNOSIS}

Initially the patient presented with non-specific acute symptoms that may be in keeping with a common viral infection (rhinoviruses, non-SARS-CoV-2 coronaviruses and influenza virus). However, with the pyrexia and cough one needs to consider community-acquired pneumonia and SARS-CoV-2. ${ }^{4}$ The lymphocytosis clearly signified a viral aetiology. 


\section{OUTCOME AND FOLLOW UP}

On the evening of day 12 , the patient felt worse and prednisone $50 \mathrm{mg}$ orally per day was initiated by the clinician. Oxygen saturation at this point was $94 \%$, pulse 101 beats $/ \mathrm{min}$, respiratory rate 44 breaths $/ \mathrm{min}$ (bpm) and blood pressure was 82/66 mmHg.

The patient returned to the A\&E unit where her respiratory rate was assessed to be $24 \mathrm{bpm}$. In view of the tachypnoea she was offered an admission bed however declined due to personal reasons.

The patient's clinical course improved overnight and on day 13, her respiratory rate was $22 \mathrm{bpm}$. On day 14 , her clinical pattern had shown improvement with a respiratory rate consistently below $20 \mathrm{bpm}$ and to the extent that the she was able to walk for the first time in days.

\section{Discussion}

The COVID-19 pandemic has created an unprecedented strain on the healthcare systems of many countries. ${ }^{5}$ At the zenith of the COVID-19 outbreak in China, there were not enough critical care beds available. $^{6}$

In Gambia, beds and personnel trained in critical care are limited to tertiary hospitals, as assessed; therefore, mortality associated with COVID-19 is likely to exceed the reported case fatality rate of $2.3 \%{ }^{7}$ This is likely in a multitude of under-resourced countries. Critically ill patients are likely to end up in general wards and those cases requiring treatment in general wards are likely to be turned away from hospital.

With hospital resources currently constrained, managing patients presenting with mild cases and who do not require high flow oxygen, or ventilation (invasive or non-invasive), with remote clinician advice may be a viable option to consider. In order to have a successful outcome, there should be a nurse or at least a competent adult on site to monitor the patient and report back to the clinician. Preferably, there should be a video link to the access the doctor remotely however in extremely under-resourced areas, a telephone link should be sufficient. ${ }^{8}$ Furthermore, there should be an ambulance service available to transfer a deteriorating patient to a hospital.

Our remote clinician-based home management protocol involved the monitoring of vital signs, oxygen via nasal cannula titrated to achieve oxygen saturation of $92 \%$ to $96 \%$, pronation for 2 hours and then a seated break for 15 to 30 minutes, Rivaroxaban $10 \mathrm{mg}$ orally 12 hourly, Prednisone at $0.75 \mathrm{mg}$ per $\mathrm{kg}$ body weight, and Vitamin C, D and Zinc supplementation. ${ }^{9}$

Pronation has been shown to be of value in improving oxygenation by reducing shunt fraction. Selfpronation has been recommended albeit with high flow nasal oxygen (HFNC). ${ }^{10}$ Slessarev et al. in 2020, reported that the prone position results in more even distribution of lung tissue leading to further uniform alveolar distribution and pulmonary perfusion and thereby improving oxygenation which prevents 
worsening of dyspnea, reduces lung oedema and progression of the disease. The authors proposed that HFNC can meet oxygen demands while allowing patients to manage their body position independently through self-proning. In addition to preserving ventilator capacity which is already restricted, routine use of HFNC combined with self-proning can be broadly applied to COVID-19 patients with hypoxemia and normal work of breathing. This care approach would be beneficial to under-resourced countries where hitech ICU techniques may not be available. ${ }^{10}$

Current evidence suggests that the coagulation cascade is significantly disrupted during SARS-CoV-2 infection and predisposes patients to thrombosis due to excessive inflammation, platelet activation, stasis and endothelial dysfunction. ${ }^{11,12}$ The case report by Di Tiano et al. (2020), highlights the risk of thrombotic complications after COVID-19 infections and supports the use of effective anti-thromobotic therapy. ${ }^{12}$ Therefore we included Rivaroxaban $10 \mathrm{mg} 12$ hourly as part of our protocol.

Chen et al. (2006) reported that the use of corticosteroid at low to moderate doses in COVID-19 patients. It was reported in a retrospective study of 401 patients, proper use of corticosteroids decreased the length of hospital stay and the mortality rate in critically-ill COVID-19 patients without causing secondary infections and other complications. ${ }^{13}$ A systematic review performed by Siemieniuk et al. in 2015, suggested that corticosteroids could reduce mortality and the need for mechanical ventilation in patients with severe community-acquire pneumonia. ${ }^{14}$

In another study performed by Wang et al. (2020), the efficacy and safety of corticosteroid usage in the treatment of COVID-19 pneumonia in 46 patients were evaluated. It was concluded that early low dose and short-term application is associated with a faster improvement of clinical symptoms and absorption of lung focus. ${ }^{15}$

The features of COVID-19 pneumonia, as reported by Gattinoni et al. (2020), are severe hypoxemia often associated with near normal respiratory system compliance, and these patients however may present differently from each other. The authors reviewed several cases (150 patients) and after further discussions with colleagues treating these patients, proposed that patients presented with different COVID-19 patterns in the emergency department and which was dependent on: the severity of the infection and comorbidities; the ventilator responsiveness of the patient to hypoxemia and the time lost between the onset of disease and observation in the hospital. ${ }^{16}$

This case report represented a mild case of COVID-19 associated pneumonia that we managed successfully without hospital admission. While we do not advocate that patients with COVID-19 associated pneumonia be managed at home routinely, it does show that managing milder cases in their homes may greatly assist in preventing the overloading of hospital capacity thereby saving those resources for the moderate and severe cases

\section{Conclusion}


The critical care capacity and resources in both public and private hospitals, in particular, ICU/High care beds and medical equipment are constrained posing a great challenge within the current COVID-19 scenario and is placing immense pressure on the provision of healthcare. In South Africa we are already experiencing our peak in certain provinces with the rest of the country set to follow within a short space of time and with the remainder of most of the African continent to follow.

Therefore, the possibility of remote clinician based home management of patients with COVID-19 associated pneumonia should be considered. Further research will likely prove that remote clinicianbased monitoring or artificial intelligence monitoring of patients on home oxygen may hold the key to preventing the collapse of healthcare systems in under-resourced countries due to the COVID-19 pandemic.

Teaching points:

- The Covid19 pandemic has resulted in a demand for hospital beds. There is a group of patients that may be managed via remote home based care.

- There is no consensus regarding protocols for home based care, we thus provide a framework that was used to successfully manage this condition. ( Table 1)

- Careful selection of patients and remote physician care may be a viable model during the covid19 pandemic.

\section{Declarations}

The Patient consented to the use of the clinical information for the purpose of the written case report.

Availability of supporting data: all data are available on request to the corresponding author, Shivesh.maharaj@wits.ac.za

Competing interests: None to declare

Funding: The study is funded by the authors

Authors' contributions: SM and NK conceived the study and SM identified the data. NK extracted the data and undertook the analyses, assisted by KH .SM prepared the initial draft the paper which both authors revised. The corresponding author attests that all listed authors meet authorship criteria and that no others meeting the criteria have been omitted.

Acknowledgements: DrF Lambert and P Patel for their assistance

\section{References}

1. Topf MC, Shenson JA, Holsinger CF, et al. Framework for prioritizing head and neck surgery during COVID-19 pandemic. Head \& Neck 2020;42:159-1167. 
2. Kasle DA, Torabi SJ, Savoca EL, et. Al. Outpatient Otolaryngogoly in the Era of COVID-19: A DataDriven Analysis of Practice Patterns. Otolaryngo Head Neck Surg. Epub ahead of print 12 May 2020. DOI: $10.1177 / 0194599820928987$.

3. Evans S, Mitchley A, Maphanga C. Coronavirus: Gauteng's private hospitals under pressure but not full - hospital groups. news24, 5 July 2020. Retrieved from:

https://www.news.com/news24/SouthAfrica/Investigations/cornovirus-gautengs-private-hospitalsunder-pressure-but-not-full-hospital-groups-20200705?isapp=true

4. Kondo Y, Miyazaki S, Yamashita R, et al. Coinfection with SARS-CoV-2 and influenza A virus. BMJ Case Rep 2020;13:e236812. Doi:10.1136/bcr-2020-236812.

5. Castro MC, de Carvalho LR, Chin T et al. Demand for hospitalization services for COVID-19 patients in Brazil. Epub ahead of print 1 April 2020. DOI:10.1101/2020.03.30.20047662.

6. Xie J, Tong Z, Guan X, et al. Critical care crisis and some recommendations during the COVID-19 epidemic in China. Intensive Care Med 2020; 46:837-840.

7. Touray S, Sanyang B, Zandrow G, et al. An assessment of critical care capacity in Gambia. J Crit Care. Epub ahead of print 18 July 2018. DOI:1016/j.jcrc.2018.07.022.

8. Greenhalgh T, Koh GC and Car J. Covid-19: a remote assessment in primary care. $B M J$ 2020;368m1182.

9. Matthay MA, Aldrich JM, Gotts JE. Treatment for severe acute respiratory distress syndrome from COVID-19. Lancet Respir Med. Epub ahead of print 20 March 2020. DOI: https://doi.org/10.1016/S2213-2600(20)30127-2.

10. Slessarev M, Cheng J, Ondrejicka $M$, et al. Patient self-proning with high-flow nasal cannula improves oxygenation in COVID-19 pneumonia. Can J Anaesth. Epub ahead of print 21 April 2020. DOI:1007/s12630-020-01661-0.

11. Bikdeli B, Madhavan MV, Jimenez D, et al. COVID-19 and thrombotic or thromboembolic disease: implications for prevention, antithrombotic therapy, and follow-up. J Am Coll Cardiol 2020;75(23):2950-2973.

12. Di Tano G, Moschini L, Loffı M, et al. Late Pulmonary Embolism after COVID-19 Pneumonia despite Adequate Rivaroxaban Treatment. Eur J Case Rep Intern Med 2020; 7(7):001790.

13. Chen RC, Tang XP, Tan SY, et al. Treatment of severe acute respiratory syndrome with glucosteroids: the Guangzhou experience. Chest 2006;129:1441-1452.

14. Siemieniuk RAC, Meade MO, Alonso-Coello P, et al. Corticosteroid therapy for patients hospitalized with community-acquired pneumonia: a systematic review and meta-analysis. Ann Intern Med 
2015;163:519.

15. Wang Y, Jiang W, He Q, et al. Early, low-dose and short term application of corticosteroid treatment in patients with severe COVID-19 pneumonia: single centre experience from Wuhan, China. MedRxiv. Epub ahead of print 12 March 2020. DOI:https://doi.org/10.1101/2020.03.06.20032342

16. Gattitoni L, Chiumello D, Caironi P, et al. COVID-19 pneumonia: different respiratory treatments for different phenotypes? Intensive Care Med 2020;46:1099-1102.

\section{Table}

Table 1: Clinician-based home management protocol for COVID-19 patients with associated pneumonia 
iteria: $\quad$ 1. Age: 18-60-year-old patients

2. C $\phi$-morbidities: should not have any co-morbidities like hypertension, diabetes, asthma, chronic obstructive pulmonary disease

3. Ability to self-isolate in a room

4. Nurse or adult supervision at home

5. A cess to clinician: regular communication via telephone or video-link is suggested every 4 hours

inical Criteria:

1. Monitoring of vital signs: temperature, blood pressure, heart rate, respiratory rate, oxygen saturation

2. Clinical exam: COVID-19 positive pneumonia confirmed by a Chest XRay

3. $\mathrm{SPO}_{2}>90 \%$ on room air (if $\mathrm{SPO}_{2}<90 \%$ on room air, transfer to hospital)

4. $R \mathbb{R}>24 / \mathrm{min}$ (if respiratory rate stays above 24 breaths per minute despite oxygen and pronation, transfer to hospital)

5. Temperature $>38^{\circ} \mathrm{C}$ (transfer to hospital if remains as such)

6. Heart rate $>100$ beats per minute (transfer to hospital if remains as such)

inical

bservation: 1. Monitor respiratory rate, oxygen saturation, blood pressure, heart rate, every 4 hours

2. Performed by nurse or a competent family member if healthcare worker is unavailable

xygen Support:

1. Maintain target $\mathrm{SPO}_{2}$ of $92 \%$ to $96 \%$ via nasal prongs @ 2 to 4 litres/min

onation:

1. Awake proning for $120 \mathrm{~min}$. Alternate with upright seating for 15 to 30 minutes.

tticoagulation:

1. Rivaroxaban $10 \mathrm{mg}$ twice daily orally

eroids:

1. Prednisone 50mg daily orally (to be used with persistent tachypnoea despite adequate $\mathrm{SPO}_{2}$ )

ipplementation

Steroids: 1. Omeprazole 20mg twice daily orally

'reventative2. Zinc 50mg twice daily orally

sses) 3. Vittamin D 50 000iu weekly orally

4. Vitamin C 500mg twice daily orally

ipport:

1. Provision of emotional and psychological support and reassurance to patient

2. Ability to communicate to family and friends via cellphone or any other suitable electronic platforms 


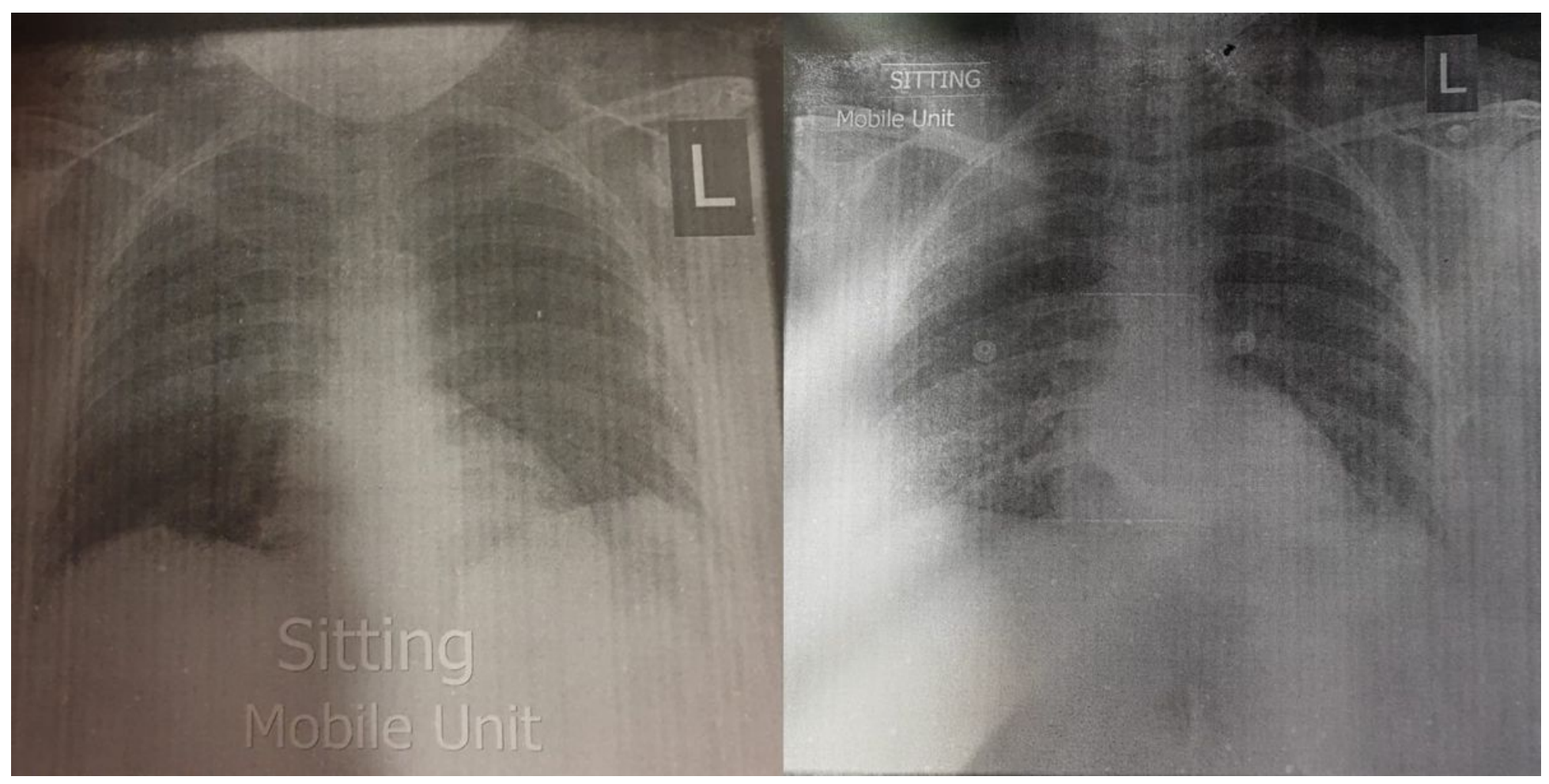

Figure 1

Left: 1a)Chest X-Ray on Day 4. Right: 1b0Chest X-Ray on Day 12 